\title{
Realizing Automatic Conversion of Service Models into Web Services towards Dynamic and Adaptive Business Process Management
}

\author{
Teshome Megersa Bekele and Weihua Zhu, Member, IACSIT
}

\begin{abstract}
The highly competitive and rapidly changing nature of the current business environment creates tremendous pressure on organizations to collaborate. This led to utterly different type of business eco-system in which individual organizations in collaborative business services increasingly demand for a collaborative business process that can easily adapt to changes. As a result, the development and management of organization's IT infrastructures that can able to cope up with the requirements of dynamic and flexible business environments are challenging. Service-based approach can enhance the development and management of collaborative processes through service abstraction to satisfy the needs of today's dynamic business environment. Service-oriented computing paradigm uses service models to represent the requirements of continuously changing business needs through service abstraction and use platform independent interaction nature of web service and its enabling available technologies for implementation. Hence, in this paper we discussed the collaborative BPM trends and concepts, technologies and proposed a generic framework to realize automatic conversion of service models into Web services in order to support dynamic and adaptive development of collaborative business process and deploy them over the internet through a web service standards platform.
\end{abstract}

Index Terms- Business Process Management, Collaborative business process, Web services, Service-oriented Architecture, Cloud Computing.

\section{INTRODUCTION}

Creating a collaborative network of trading partners to reduce costs, shorten business process cycle times and streamline workflow is critical to business competitiveness and growth [29]. Accordingly, business world motivated businesses around the world to pay more attention to process management and process automation to improve their organizational efficiency and effectiveness. This has led to Business Process Management (BPM) being increasingly recognized as a critical factor in business success. BPM is a holistic management approach that promotes business effectiveness and efficiency while striving for innovation, flexibility, and integration with technology.

Many researchers in business vendors, research and academia institutions followed various approaches to build and deploy business process management. Traditional

Manuscript received July 14, 2011; revised August 10, 2011.

Teshome Megersa Bekele is with Computer Science at the Institute of Technology of Arba Minch University, Ethiopia.

Weihua Zhu is with college of Software Engineering of Chongqing University, China. approaches to building and implementing BPM systems have used workflow technologies to design and control the business process. Workflow-based systems follow highly structured and predefined workflow models; they are well suited to applications with standard inputs, processes, and outputs. This type of BPM is typically delivered with an integrated set of tools that provide a closed-loop system for optimizing business performance [33].

However, Contemporary business processes are becoming increasingly complex and dynamic as they seek to cope with a wide range of internal and external interactions and changes. Modern BPM expands to cover the partner organizations' business processes across organizational boundaries supports organizations' to coordinate the flow of information among organizations' and link their business processes. Accordingly, business environments have been changing from centralized-and-closed to distributed-and-open. The processes usually evolve and change over time due to complex interactions, resource competition, breakdowns and abnormal events, and other sources of uncertainty. A business process also displays complexity as a result of multiple interactions of its internal components and interaction between the process and its environment.

Service-oriented computing is an emerging paradigm to integrate processes in the collaborative environment which encompasses business processes of multiple organizations. It provides seamless and loosely-coupled, technology neutral and location transparency supporting business collaborations. Web services provide the basis for the development and execution of business processes that are distributed over the network and available via standard interfaces and protocols. This is due to the fact web services are platform-neutral, so as long as they comply with the common standard they can be integrated into a more complex structure. Web services also supports the execution of short-running transactions within an organization (similar to traditional distributed transactions) and long-running transactions across organizations. Combined with recent developments in the area of distributed systems, workflow management systems, business protocols and languages, services can provide the automated support needed for e-business collaboration and integration both at the data and business logic level. They also provide a sound support framework for developing complex business transaction sequences and business collaboration applications [32].

Nonetheless, the current WS collaborative business process enabling technologies like BPEL lacks a capability to accommodate the needs of current dynamic and flexible every changing business world. To use the advantage of Web 
Services technology that can connect incompatible stand-alone systems to integrate a complex distributed system needs a holistic approach that address the requirements of collaborating businesses in all perspectives of corporate enterprises. The research works of [2] described such an approach.

However, as today's business processes are internet-mediated, there should be a way to tie these service models into web services and create web services standard solutions in order to develop collaborative business supported over the internet. Therefore, the aim of this research is to support development of dynamic and flexible collaborative BPM with generic framework for automatic conversion of services models (rule-based service abstraction) into web services standards to execute them. This in turn create a platform to enable corporate enterprises (businesses) to achieve flexible and adaptive collaborative business process management and development both at design and run-time independent of specific services orienting computing.

The remaining section of the paper discusses background concepts, dynamic and flexible business collaboration development trends, and collaborative BPM emerging technologies, Services models into Webservices conversion development and conclusion and future research directions.

\section{BACKGROUND CONCEPTS}

\section{A. Business Process}

The concept of business process goes back to Adam Smith and the origins of economic theories. However, the concept was adopted and exploited in areas outside manufacturing only in the past 20 years, thanks to the rise of a cluster of IT-based technologies that could support business process automation, optimization and reengineering.

Today, business processes constitute the centerpiece around which organizations structure their operations, measure their performance, and base their competitiveness. Business processes have also become a core concept for organizational information systems in the sense that they define the operational environment and many of the requirements for such systems [30]. A business process can be simply defined as a collection of activities that create value by transforming inputs into more valuable outputs. These activities consist of a series of steps performed by actors to produce a product or service for the customer. In more details, a business process is typically a coordinated and logically sequenced set of work activities and associated resources that produce something of value to a customer [16]. Each process has an identified customer; it is initiated by a process trigger or a business event (usually a request for product or service arriving from the process customer); and it produces a process outcome (the product or a service requested by the customer) as its deliverable to the process customer.

Given the scope and variety of actions that are needed to produce the product or service, the process is differentiated (sub-divided) into a set of tasks or activities. These tasks are assigned to and performed by actors (either machines or humans), using resources such as work-stations; machines; raw materials; and supplies that are available to the actors[6] .Business process falls under management processes enable organizations to discuss and reason about the purpose and high level objectives of their business( e.g. corporate governance and strategic management), operational processes allow organizations to consider and examine how their day-to-day businesses activities help achieve the objectives set up at the strategic level (e.g. purchasing, manufacturing, advertising and marketing, and sales) or supporting processes (service level processes) empowers organizations to capture and investigate how their IT infrastructure provides support for the operational activities within the organizations, across cooperating organizations (e.g. Accounting, Recruitment, Call center, Technical support)[4].

A business process begins with a mission objective and ends with achievement of the business objective. The analysis of business processes typically includes the mapping of processes and sub-processes down to activity level. Business Processes are designed to add value for the customer and should not include unnecessary activities. The outcome of a well designed business process is increased effectiveness (value for the customer) and increased efficiency (less costs for the company).

\section{B. Business Process Management}

Nowadays, in continuously changing business environment, competitive advantage of organizations depends on ability to excel in key business processes. As a result, businesses around the world are paying more attention to process management and process automation to improve organizational efficiency and effectiveness. This has led to business process management being increasingly recognized as a critical factor in business success. Business process management (BPM) is a management approach focused on aligning all aspects of an organization with the wants and needs of clients. It is a holistic management approach that promotes business effectiveness and efficiency while striving for innovation, flexibility, and integration with technology.

In other words, business process management refers to activities performed by organizations to design (capture processes and document their design in terms of process maps), model (define business processes in a computer language), execute (develop software that enables the process), monitor (track individual processes for performance measurement), and optimize (retrieve process performance for improvement) operational business processes by using a combination of models, methods, techniques, and tools [37]. It is argued that BPM enables organizations to be more efficient, more effective and more capable of change than a functionally focused, traditional hierarchical management approach. An empirical study [24] reveals that BPM helps organizations to gain higher customer satisfaction, product quality, delivery speed and time-to-market speed.

Among various approaches or methods for BPM, today's BPM solution focuses on the use of information technologies to support or automate business processes, in whole or in part, by providing computer-based systems support. These technology-based systems help coordinate and streamline business transactions, reduce operational costs, and promote 
real-time visibility in business performance. Based on the definition of the business process, it is possible to define the term business process management, which includes concepts, methods, and techniques to support the design, administration, configuration, enactment and analysis of business processes. These management activities can be can be arranged in a life cycle, which consists of phases that are related to each other based on their logical dependency. Because of space limitation we didn't include the phases and their details. (See [3])

\section{DynAmiC AND FleXible Collaborative Business PROCESS MANAGEMENT}

Today's business world requires a smart collaboration. This is so because the nature of competition amongst businesses has changed significantly. The core change has resulted from the advances of electronic communications. The ability of businesses to execute their business processes independent of geographical or technological boundaries has brought about an utterly different type of business eco-system. As a result organizations are facing rapidly changing market conditions, new competitive pressures, new regulatory fiats that demand compliance, and new competitive threats. All of these circumstances drive the need for the IT infrastructure of an organization to react in support of these business requirements. This in turn led to an increasing demand from individual organizations for corporate business services that can easily adapt to changes [2].

According to research on BPM maturity, the effective collaboration with the external stakeholders of an organization (such as customers, suppliers, or distributors) in its BPM projects is an integral characteristic of organizations with high BPM maturity, of "intelligent operating networks" [18]. The stimulation, the management, and the exploitation of BPM networks and collaboration can be regarded as a key challenge to the practice of organizational BPM. Also [8] argues that organizations need to explore new pathways to systematically make use of know-how that lies outside of an organization's boundaries through new forms of collaboration.

Many researchers in business vendors, research and academia institutions followed various approaches to build and deploy business process management. Traditional approaches to building and implementing BPM systems have used workflow technologies to design and control the business process. Workflow systems are managed document processes where people executed the workflow steps of process. Workflow-based systems follow highly structured and predefined workflow models; they are well suited to applications with standard inputs, processes, and outputs. This type of BPM is typically delivered with an integrated set of tools that provide a closed-loop system for optimizing business performance [8].

On other hand, today's BPM systems manage processes that include person-to-person works steps, systems-to-systems communications or combinations of both. As a result contemporary business processes are becoming increasingly complex and dynamic as they seek to cope with a wide range of internal and external interactions and changes.
Contemporary BPM expands to cover the partner organizations' business processes across organizational boundaries supports organizations' to coordinate the flow of information among organizations' and link their business processes. Accordingly, business environments have been changing from centralized-and-closed to distributed-and-open.

Business processes usually evolve and change over time due to complex interactions, resource competition, breakdowns and abnormal events, and other sources of uncertainty. In other words, business process displays complexity as a result of increased changes and interactions arising from turbulent environments, needs for creating and maintaining a fit between the requirements and supporting technologies for process management, needs for integrating technological, organizational, and managerial perspectives into process management, and managing complex and interactive processes across multiple organizations. In general, a business process displays complexity as a result of multiple interactions of its internal components and interaction between the process and its environment. As a result, a traditional approaches and technologies for process management are often inadequate for complex and dynamic situations due to lack of flexibility and adaptability [38].

Accordingly, BPM should be able to manage a number of components and their complex interactions in business processes, in particular in continuously changing and interplayed environment. Furthermore, attention should be paid to situations where dynamic collaboration and soft-connection between business partners is playing increasingly important role complex dynamic systems that adapt to continuously changing and unpredictable environments in order to survive. To manage complexity and foster flexibility and adaptability of BPM, a number of researchers have been investigating the approaches and techniques for developing BPM systems for an increasingly turbulent environment [9]. Most studies have focused on present process structures and provide rapid response to changes that lead to temporary and short term fluctuations in the organization's activities.

However, in recent years several works have been done to meet today's business needs. The development trends of collaborative BPM based on research works reviewed clearly shows works from extended workflow to handle adaptive and dynamic business requirements [15], building virtual organizations alliances by giving autonomy for each participating organizations [31], developing and governing collaborations models based on rules and model-driven development [2], service-oriented computing, web services technologies and agent-oriented computing [42] to cope with current dynamic and every changing business environments. We believe that effective collaborative BPM development needs much effort from business vendors, research and academic institutions.

\section{EMERGING TECHNOLOGIES FOR BUSINESS COLLABORATIONS}

\section{A. Web services and Collaboration}

Web services technologies are emerging as a powerful 
vehicle for organizations that need to integrate their applications within and across organizational boundaries. Web services can be seen as a way to design seamless and flexible interaction across applications within and across firm boundaries. A Web Service, defined by W3C (World Wide Web Consortium [40], as a software application identified by a URI (Uniform Resource Identifier), whose interfaces and bindings are capable of being defined, described, and discovered by XML, and which supports direct interactions with other software applications using XML-based messages via Internet-based protocols.

In other words, web service is a self-describing, self-contained software module available via a network, such as the Internet, which completes tasks, solves problems, or conducts transactions on behalf of a user or application. Web services constitute a distributed computer infrastructure, made up of many different interacting application modules trying to communicate over private or public networks to virtually form a single logical system. It can be viewed as a natural evolution of the Web and it will facilitate complex business and science application interactions [12]. Web Services identifies global elements of the global Web Services network that are required in order to ensure interoperability between Web Services and delivery mechanism that can serve many different consumers on many different platforms at the same time. Web Services technology act as an enabler to connect incompatible stand-alone systems to integrate a complex distributed system in a way that was not possible with previous technologies [36]. They enable Web applications to "talk" with each other independent of their technical environments which could be defined as open standard (XML, and SOAP)-based Web applications that interact with other Web applications for the purpose of exchanging data.

The collaborative coordination of business processes through WS includes the execution of short-running transactions within an organization (similar to traditional distributed transactions) and long-running transactions across organizations. XML Web Services are the fundamental building blocks that provide exchange of information over different computing standards on the Internet. XML uses open standards to communicate and collaborate amongst applications. Thus we see that WS tend to offer opportunities that are way beyond the commonly discussed business integration - that merely deals with document exchanges. Web Services hold the promise of considerable gains for many organizations by giving them the opportunity to develop techniques to effectively integrate disparate applications by leveraging computer technology [23]. The above statement emphasizes that not only can integrated systems provide better business value by sharing data, communicating results and improving overall functionality, but the ability to integrate systems also opens up doors to synergies between systems of disparate organizations.

One of the challenges of the collaboration occurs when the involved organizations have to make the investment necessary for replacing redundant or older systems as well as building a dynamic platform that incorporates multiple standards [19]. The challenge is further increased when, in unstructured e-collaboration (without prior contract), creating or exchanging of non-standard documents takes place. The ability to promote as well as locate services, however, is provided through Universal Data Dictionary Integration (UDDI). It is a platform-independent, XML-based registry, allowing businesses worldwide to list themselves on the Internet. The UDDI specifications provide a mechanism to register and locate WS. It defines an electronic business registry in which businesses can describe their business and register their WS as well as discover and integrate with other businesses that offer Web Services [35].

Web Services are application components each of which normally performs a single discrete functionality of the overall application. Orchestration and choreography are the two emerging standards for creating business processes from multiple Web Services. Orchestration refers to an executable business process that can interact with both internal and external Web Services while choreography is more collaborative and allows each involved party to describe its part in the interaction [10].

In general, recent development trends and the platform independent service delivery nature of WS and research works to enhance process-based composition of services promote its importance for process integration. As a result, WS is one of an emerging and a promising approach to automate business process within and across organizational boundaries. For instance, a rule inference framework called DYflow provides a mechanism for end users to define their business objectives or goals and dynamically composes Web services to execute business processes [36]. And other similar works revealed that WS has a potential for designing collaborative BPM.

\section{B. Service Oriented Architecture (SOA) and Collaboration}

Service-oriented computing (SOC) is an emerging cross-disciplinary paradigm for distributed computing that is changing the way software applications are designed, architected, delivered and consumed. It is intended to facilitate business collaboration and application integration at a global scale. It is a computing paradigm that uses services as fundamental elements of the development process of business applications. To build the service model, SOC relies on the Service Oriented Architecture (SOA), which is a way of reorganizing software applications and infrastructure into a set of interacting services [28].

Service-oriented architecture is an approach to organizing software in the form of independent, interoperable services that can be composed and recomposed to fulfill multiple business requirements. It is an emerging paradigm for architecting and implementing business collaborations within and across organizational boundaries. SOA enables seamless and flexible integration of Web services or applications over the Internet. It supports universal interoperability and location transparency. It also reduces complexity of business applications in large-scale and open environments by providing flexibility through service-based abstraction of organizational applications [26].

Services, which are the heart of service-oriented computing, are autonomous, platform-independent 
computational elements that can be described, formally specified, published, discovered, orchestrated and programmed using standard protocols for the purpose of developing massively distributed interoperable applications organized in the form of a network of collaborative components functioning within or across organization borders [22]. A service is a mechanism to enable access to a set of one or more capabilities, where the access is provided using a prescribed interface and is exercised consistent with constraints and policies as specified by the service description.

The highly competitive nature of the current business environment creates tremendous pressure for organizations to collaborate. It is essential for companies to understand rapidly changing business circumstances. The rapidly changing environment encourages enterprises to integrate their business functions into a system that efficiently utilizes ICT. The recommended implementation of the integration utilizes the technologies of SOA to integration processes in the collaborative environment of the business processes of multiple organizations.

Though the industry has made much progress in foundational areas, it is still a highly dynamic market. There is currently a lack of strong support for a standard way of working with processes that involve human-interactions, even though there are recommendations to address them. BPEL currently does not handle person-to-person processes, so an extension has been proposed called BPEL for People (BPEL4People) [27]. Similar capabilities can also be found in XML Process Definition Language (XPDL).

The security issues are about message integrity, confidentiality and authentication. In SOAP-based communication, XML extensions are created to support the use of multiple security tokens (such as username, certificates, (Security Assertions Markup Language) SAML) within the message. The example of such effort comes from the WS-Security specification. Communication standards are a priority given the need to handle communication problems gracefully between any two systems (due to, say, a network or application outage). In SOAP-based Web-service integration, the two competing standards, backed by different vendors, are WS-ReliableMessaging (WS-RM) and WS Reliability (WS-R).

One other area in flux involves how transactions are managed during system-to-system interactions with Web services. BPEL (Business Process Execution Language) workflow transactions, for example, can be short or long running. WS-Transaction and WS-Coordination services are ways of supporting transactional Web services. While another standard, BTP (Business Transaction Protocol) supports similar needs, currently BPEL and WS-* extensions are getting more visibility. In this regards a lot expected from researchers, academia and industry to integrate them and come up with a better technology to develop SOA-enabled collaboration that deal with the fast occurring change of business worlds.

\section{Cloud Computing and Collaboration}

Cloud computing has become a significant technology trend, and many experts expect that cloud computing will reshape information technology (IT) processes and the IT marketplace [7]. It is changing the fundamental way in which information technology services are being delivered. It is also spawning a whole new series of terms and acronyms like Software-as-a-Service (SaaS), Platform-as-a-Service (PaaS), Infrastructure-as-a-Service (IaaS), Business Operations Platform (BOP), Public Cloud and Private Cloud.[17] It seems as though new terms are being coined every day. As both established and emerging vendors move into the Cloud Computing marketplace the definition of Cloud Computing continues to morph. But one thing seems to remain rather constant and that is the delivery of "services." The Cloud services concept centers around the idea that information technology applications are being delivered to the face of a web browser from an amorphous "cloud" - generally referring to the Internet - outside the typical data-driven, on-premise computing architectures in common use today. This emphasis on services delivery targets the commodity (a service) being delivered, and de-emphasizes the method or infrastructure behind how that service is delivered.

Cloud computing can be defined as a new style of computing in which dynamically scalable and often virtualized resources are provided as a services over the Internet. Advantages of the cloud computing technology include cost savings, high availability, and easy scalability. It has become a great solution for providing a flexible, on-demand, and dynamically scalable computing infrastructure for many applications [7].

Cloud computing categorized as public cloud (or external cloud) in which computing resources are dynamically provisioned over the Internet via Web applications or Web services from an off-site third-party provider, private cloud (or internal cloud) built for the exclusive use of one client, providing full control over data, security, and quality of service and hybrid cloud which combines multiple public and private cloud models. Hybrid clouds introduce the complexity of determining how to distribute applications across both a public and private cloud [13].

The likely natural evolution of Cloud Computing is the formation of safe and efficient Cloud "ecosystems" where applications and services can be created and exchanged in a known, secure, high-performance computing environment. Cloud enabling technologies like web services, workflows and others are those already extensively used in business process management development. As a result cloud-enabled Business Process Management systems are the perfect tool for hosting these ecosystems and as such will become the de facto "operating systems" of Cloud Computing. BPM Suites (BPMS) can orchestrate web services - major building blocks for Cloud applications, allow collaboration between workers, systems and information across organizations and geographies, administer business rules, provide real-time feedback about work-in-progress, monitor business outcomes against performance targets and continuously refine and adjust process flows to optimize performance [20].

A BPM system running on the appropriate Cloud-connected hardware configuration makes the ideal Platform-as-a-Service ecosystem because the BPM system itself provides structure, security and consistent rules across process, user, geographic and organizational boundaries less 
Wild West and more civilization. And cloud-delivered BPM with minimal or non-existent start-up costs coupled with low monthly subscription pricing and libraries of pre-built applications will make the power of Business Process Management available and affordable to every organization. It will also allow organizations and individuals to begin learning about and working with process modeling and process discovery in an inexpensive, risk-free and productive environment.

\section{RELATED WORKS}

Contemporary business processes are becoming increasingly complex and dynamic as they seek to cope with a wide range of internal and external interactions and changes. As we described collaborative BPM development trends in section III developing and deploying collaborative BPM needs a thorough understanding of today's business needs, organizational structure and policies, integrating new development approaches with existing IT infrastructure to comply with organizations strategy, a clear separation of functional and technical requirements, and a way to manage processes across multiple organizations. Accordingly, seamless integration and loose coupling interactions of heterogeneous systems development requires new approaches both at design and run-time to fulfill the need of continuous and ever changing business needs.

In order to deliver the adaptive business services demanded by today's agile and dynamic business climate, different approaches have been explored. Among these approaches traditional workflow can be seen as a major move to design BPM at the beginning. Nevertheless, the predefined nature of traditional workflow hindered it to address the need of modern business environment [39]. In addition, an attempt to build flexibility and adaptability to workflow is not able to fully cater the needs of business process needs. This is due to the fact that changes in processes enforced to design time [11]. Current solutions such as BPEL4WS [14] and those specified by ebXML are pre-determined and pre-specified, have narrow applicability and are almost impossible to reuse and manage.

On the other hand, in the area of service composition the recent success to develop and manage complex and dynamic business processes came up with the concepts of service technology. Besides, the rise of Internet-mediated e-Business brings the era of demand-driven and resource-based soft connections of business organizations. A business process can be dynamically established by connecting or composing services provided by different organizations. Service-oriented architecture provides a real platform for resource selection and allocation in order to implement seamless and flexible integration of business processes over the Internet. Web services are a key technology to enable SOA implementation. [24] in their works indicated how service can composed and enable to support universal interoperability and location transparency. It also reduces complexity of business applications in large-scale and open environments by providing flexibility through service-based abstraction of organizational applications.

However, a holistic and systematic way to develop and manage business process required from the perspective of the requirements of modern business environment in which the current service technology doesn't fulfill some of them in this regards. The research works of [2] described such an approach in which Business Collaboration Context Framework (BCCF) used to modularize the business collaboration context by adopting a three dimensional view being aspect, level and part, and Model-Driven Development (MDD) was used to describe the business collaboration context, and a rule-based approach for developing business collaboration models in which rules used to help the designers to rapidly effectuate changes while at the same time manage consistency for each partner in the collaboration as well as consistency for the collaboration as a whole. However, their research works lack of a mechanism to tie the services models with web services to execute them.

In general, defining and implementing flexible and adaptive business processes supported by flexible Information Technology (IT) systems is of significance to organizations because this would allow them to collaborate with partners in new ways resulting in inimitability of the processes, and this in turn allows them to adapt to the changing environment. Accordingly, to enjoy the advantage of web services we need a generic system to convert service models based on rule-based service models design techniques into web services standard solutions to deploy models over the internet.

\section{CONVERSION FRAMEWORK DEVELOPMENT}

\section{A. Service Models Development Approaches}

Nowadays there are various approaches to develop collaboration models. In this paper we present the ruled-based collaboration models development that we use to construct service models.

1) Business Collaboration Context Framework (BCCF)

The BCCF captures the context in which business collaboration development and management takes place by adopting a three dimensional view, being aspect, level and facet. Through this three dimensional view modularization of the definition and management of business collaborations is achieved, as such reducing the inherent complexity of collaborations. The aspect dimension, places emphasis on observable behavior between participants depicting how they are expected to behave, exposed behavior of an individual participant defining how it can behave, and internal behavior of an individual participant specifying its private activities [34].The level dimension expresses that collaboration behaviors can be observed as the strategic level focusing on purpose and high level requirements; operational level specifying operational activities supporting achievement of these requirements; and service level concentrating on the realization of these operational activities via the usage of the services delivered by the IT-infrastructure [21].The facet dimension depicts the elements in a business collaboration behavior that have different contexts when observed from different levels. Five facets are distinguished what, how, who, where and when facets emphasizing a structural, functional, participation, geographical and temporal point of view respectively. The facets provide a complete coverage for each aspect at an individual level; where their semantics are 
dependent on the specific aspect and level that they describe [21] [1].

\section{2) Rule Driven Business Management}

However, the model development process will be too tiresome and complex for enterprises to do manually; especially when they want to be able to verify and validate the alignment and conformance of the models developed. Therefore, rule driven mechanism is proposed where management rules used to drive the design of models to achieve flexibility and adaptability while construction rules to verify and maintain the validity, alignment, and compatibility of designed models [2].

Management rules express the uniqueness, originality and values of individual enterprises. Management rules are specific to particular business collaborations, and can be categorized along the three dimensions in BCCF. Example rules are "All customers with status 'golden' get a $30 \%$ discount”. Interpreted in the context of modeling, management rules represent all business collaboration requirements that are likely to be subject to change. By externalizing these requirements the design of models is made flexible as rules can be administered to incorporate dynamic requirements. Furthermore, enterprises can chain these rules and use them for making complex decisions and diagnoses; as well as tailor them to handle different scenarios. Business collaboration is also made adaptable as enterprises can manage changes to collaboration designs with minimum disruption by redefinition of their rules.

Construction rules reflect knowledge about the business collaboration domain. They are generic in nature, that is, applicable to all business collaborations. Construction rules can be found throughout the business collaboration context just like management rules. Examples include that "each step must be decomposed into at least one task", "two tasks cannot be dependent on each other”, and "a mapping from a task requiring authentication to an operation not capable of doing so is not feasible. Construction rules are used to govern the development and management of business collaboration models. The construction rules define the boundaries in which models can be developed and managed, whereas such they can be employed to design models in a valid, aligned and compatible manner. That is, enterprises are able to verify that their designs are complete and correct (i.e. syntactically sound), and consistent (i.e. semantically sound). In addition, this knowledge can be updated to incorporate changes, or expanded to include new details.

Through enforcement of construction rules during design enterprises are able to develop and manage the specification of modeling description atoms in a valid, aligned and compatible manner. This allows them to create more complex structures by combining atoms, where their validity, alignment and compatibility can be ensured. As such, enterprises can employ a single mechanism at their disposal to design and manage business collaboration designs in a flexible and adaptive manner; while at the same time they are assured that these designs are valid, aligned and compatible.

\section{B. Proposed Framework Architecture}

The framework has two parts: Model development manager and the service composition manager.

\section{1) Model development Manager}

The Model Development Manager handles the process of creating models and policies from business collaborations requirements and analyzing the consistence of the policies and models created with respect to the requirements specified and then storing the results. The Model Development part of the framework has four components: policy editor, policy and rule analyzer, services model generator and repositories. The Policy editor component provides a mechanism for development manager to get requirements and rules governing the business from stakeholders in collaboration. The Policy editor passes the requirements into the policy and rule analyzer. The Policy and Rule Analyzer offers functionality for analyzing policies with regard to missing, inconsistent and redundant rules, simulate models based on their policies conforming to particular circumstances, and effectuate changes to policies in existing models. Once the problem has been solved the policy and rule analyzer passes the information to Service Model Generator. The model generator generates models and provides a mechanism to simulate and verify that policies lead consistent and coherent models and mapping under different circumstances. The simulation and verification can be done through developing a rule engine analyzer using technologies like OO jDREW rule engine to reason with non-monotonic and prioritization, usage of alternatives, and status of rules and alternatives.

Finally, the models generated and policy defined stored into Repositories component. The repository components provides policy and model representation, storage and manipulation components that provide support for creating, editing and storing policies for business collaboration models as well as generated models in the form of easily exchangeable XML based representations.

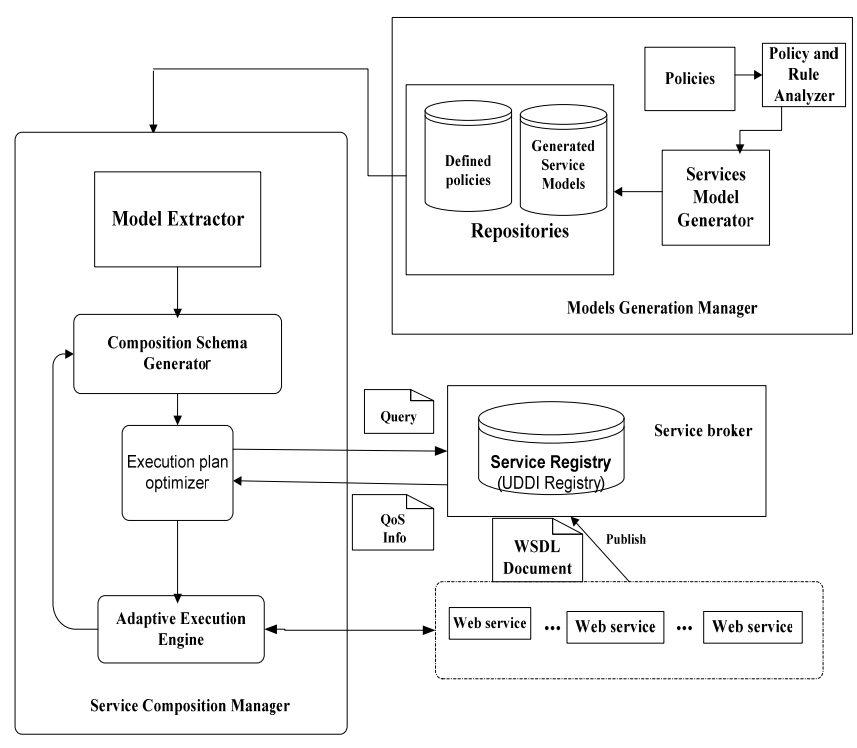

Fig. 1.Conversion Framework

\section{2) Service Composition Manager}

The Service Composition Manager component of the framework provides where the collaboration models created and policies defined extract through Model Extractor and pass onto composition schema generator to satisfy the conversion of service models into web service standards and composing them. 


\section{a) Model Extractor}

The Model Extractor component provides a mechanism to retrieve the requirements within service models and policy defined and categorizes them into business objectives description and rules governing the given services interaction with corresponding services. As explained under the Model Development Manager, the policy defined and models created are represented by simple XML. The Model Extractor retrieve these XML representation and get the business collaborations objective like services description, rules govern the interaction services with others and message passing like vertical mapping between models or horizontal mapping. The process of identifying the description of each services and rules within the collaborations models based on the policy defined initially (for detail information refers to [3]). Following this Model Extractor defines these description (services description and rule) using simple XML format. And then passes information to Composition Schema Generator.

\section{b) Service Broker}

There is a meta-data repository in service broker called service repository. We recommend the UDDI registry to implement meta-data repository. The service broker provides an interface for service repository: the publish interface and the search interface. For the service repository, the publish interface allows service providers to publish or advertise their service descriptions in WSDL. While the search interface allows the composition manager to discover Web services by service class name, operation name, input and output data [41].

\section{c) Composition Schema Manager}

The composition schema generator receives the business requirements and rules govern them from Model Extractor as input. It discovers associated business rules based on policies defined during collaboration model construction and the business objective to generate composition schemas.

The composition schema generator implements the three phase's inference algorithms described in [25] to dynamically generate one or more composition schemas which are represented internally as XML documents. For the generated composition schema, the Execution Plan Optimizer will create an optimal execution plan based on Web services' QoS information and composition schema quality information. The optimal execution plan from the set of all composition schemas will be passed to the adaptive execution engine to execute composite services. The execution engine manages the composite service's lifecycle and enables service collaboration and monitoring while executing the tasks. The execution results are used as input for the composition schema generator to refine the composition schema and the execution of composite services.

\section{Analysis of proposed Framework}

The feasibility of the framework was stated in the form of car damage insurance claiming scenarios. (See for details [3]). The development of business collaborations governed by rules will enable the collaborating business to define their business requirements in a dynamic way to fulfill the every changing business needs and stay competitive. The framework we proposed takes the generate models input and extract the Meta information of the models into business description and rules. And then Composition Schema Generator takes these information and feeds to the optimizer which creates the services and store into UDDI using XML.

In general, the approach we proposed enables dynamicity and flexibility both at design and run-time. The design time dynamicity is derived from the fact that the model generator can enable the corporate businesses to design their collaboration models, analyze the models and reinforce changes at any time based on rules. Model Extractor retrieves the policies (business requirements) and rules govern them and feed to Composition Schema Generator. As a result, the run-time adaptability in collaborations can be handled by the Service Composition Manager. In general, the proposed framework enhances techniques for the development of dynamic and flexible business process collaborations management through realizing automatic conversions of service models into web services standards to execute them independent of underlying technologies used in corporate business.

\section{CONCLUSION AND FUTURE RESEARCH DiRECTIONS}

With collaborative business processes, organizations' can create dynamic and flexible collaborations to synergistically adapt to the changing conditions, and stay competitive in the global market. In this paper we presented a research work which aims at thoroughly studying current business collaborations trends, and developing a framework to realize automatic conversion of service models (rule based service abstraction) into web service standards in order to support development of dynamic and flexible collaborative BPM both at design and run-time independent of underlying service-oriented computing. Accordingly, collaborative BPM basic concepts and development trends, and emerging technologies for enabling business collaboration and proposed a generic framework details were discussed. In general, we analyzed collaborative business process management development concepts and technologies as well as clearly showed their importance.

Work for future research will focus on examining the emerging technologies in the context of business process management and figure out how to integrate with the current development trends. For instance, Cloud computing is definitely a type of computing paradigm/architecture that will remain for a long time to come. This work will benefit professionals, researchers, and practitioners by advanced analysis and theoretical investigations of problems and solutions in developing solutions for complex BPM.

\section{REFERENCES}

[1] A. Scheer (1992). Architecture for Integrated Information Systems Foundations of Enterprise Modeling, Springer-Verlag New York, Secaucus, NJ, USA.

[2] Bart Orriens, Jian Yang (2007). Rule-based business collaboration development and management, International Journal of Business Process Integration and Management 2(3), pp. $203-214$

[3] Bekele Teshome Megersa. (2011),.Automatic Conversion of Service Models into Web Services towards Dynamic and Flexible Collaborative B PM Development, unpublished Master's Thesis, Chongqing University 
[4] “BusinessProcessManagement”,http://en.wikipedia.org/wiki/Business _process_management (collected15/11/2010)

[5] C. Peltz (2003). Web services orchestration: a review of emerging technologies, tools, and standards, Hewlett Packard White Paper, January 2003

[6] Casati, F., Ceri, S., Paraboschi, S., \& Pozzi, G. (1999). Specification and implementation of exceptions in workflow management systems. ACM Transactions on Database Systems, 24(3), 405-451.doi:10.1145/328939.328996

[7] Chappell, D. (August 2008). A short introduction to cloud platforms: An enterprise-oriented view.San Francisco, CA: Chappel and Associates

[8] Chesbrough, H. (2003). Open Innovation - The New Imperative for Creating and Profiting from Technology. Harvard Business School Press, Boston, USA.

[9] Chiu, D. K. W., Li, Q., \& Karlapalem, K. (1999). A meta modeling approach for workflow management system supporting exception handling. Information Systems, 24(2), 159-184. doi:10.1016/S03064379(99)00010-1

[10] Chris Peltz (2003).Web Services Orchestration and Choreography,(2003) .Computer, vol. 36, no. 10, pp. 46-52, doi:10.1109/MC.2003.1236471

[11] Christophides, V., Hull, R., Kumar, A., \& Simeon, J. (2000). Workflow mediation using VorteXML. Bulletin of the IEEE Computer Society Technical Committee on Data Engineering, 24(1), 40-45

[12] Chrysostomos Zeginis, Dimitris Plexousakis (2010).Web Service Adaptation: State of the art and Research Challenges, Institute of Computer Science, FORTH-ICS Technical Report 410, ICS-FORTH

[13] “CloudComputing,”Wikipedia, http://en.wikipedia.org/wiki/Cloud_computing

[14] Curbera, F., Goland, Y., Klein, J., Leyman, F., Roller, D., Thatte, S., et al. (July, 2002). Business process execution language for Web service

[15] Dickson K.W. Chiu, Thomas Trojer, Hua Hu, Yi Zhuang, Patrick C.K.Huang (2010). Flow Based Adaptive Information Integration, In the In Handbook of Research on Complex Dynamic Process Management: Techniques for Adaptability in Turbulent Environments, ed. Minhong Wang, and Zhaohao Sun, 209-231(2010)

[16] El Sawy, O. A. (2001). Redesigning enterprise processes for e-business. Boston: Irwin/McGraw- Hill Hammer, M., \& Champy, J. (1993). Reengineering the corporation: A manifesto for business revolution. London: Brealey

[17] Fingar, Peter, Dot Cloud: The 21st Century Business Platform Built on Cloud Computing, Meghan-Kiffer Press, 2009

[18] Fisher, D. M. (2004). The Business Process Maturity Model. A Practical Approach for Identifying Opportunities for Optimization. BPTrends, 9 (4)

[19] Florian Daniel, Barbara Pernici (2006). Insights into Web Service Orchestration and Choreography, International Journal of E-Business Research,2(1), pp.58-77, doi: 10.4018/jebr.2006010104

[20] George M. Barlow(September 2009), Business Process Management and Cloud Computing, A white Paper, Cloud Harbor,Inc

[21] J .A. Zachman (1987), A framework for information systems architecture, IBM Systems Journal, Vol. 26, no. 3, pp. 276-292

[22] Jana Koehler, Gustavo( 2007). Introduction to Special Theme: Service-Oriented Computing, ERCIM News , N.70, ERCM EEIG

[23] Kirda, E., M. Jazayeri, C. Kerer, and M. Schranz (2001). Experiences in engineering flexible Web service. IEEE MultiMedia , 8(1), 58-65. doi:10.1109/93.923954

[24] Kohlbacher, M. (2009): The Effects of Process Orientation on Customer Satisfaction, Product Quality and Time-Based Performance. 29th International Conference of the Strategic Management Society, Washington DC, October 11-14, http://dc.strategicmanagement.net/

[25] Liangzhao Zeng, Anne H.H. Ngu, Boualem Benatallah, Rodion Podorozhny, Hui Lie (2008). Dynamic composition and optimization of Web services. Distributed Parallel Databases, 24, 45-72. DOI $10.1007 / \mathrm{s} 10619-008-7030-7$

[26] Marjanovic, O. (2006). BPM - bridging the gap between business processes and technologies for process management. In Proceedings of the ACIS

[27] Matthias Kloppmann, Dieter Koenig, Frank Leymann, Gerhard Pfau, Alan Rickayzen, Claus von Riegen, Patrick Schmidt, Ivana Trickovic (2005), WS-BPEL Extension for People - BPEL4People ,A Joint White Paper by IBM and SAP

[28] Mike P. Papazoglou(2003). Service -Oriented Computing: Concepts, Characteristics and Directions, Web Information Systems Engineering, International Conference on In Proceedings of the 7th International Conference on Properties and Applications of Dielectric Materials (Cat.
No.03CH37417), (2003), pp. 3-12. doi:10.1109/WISE.2003. 1254461 Key: citeulike:276496

[29] Minder Chen, Dongsong Zhang, Lina Zhou (2007). Empowering collaborative commerce with Web services enabled business process management systems. Decision Support Systems,43, 530- 546.

[30] Minhong Wang, Zhaohao Sun(2009). Challenges and Solutions for Complex Business Process Management, Handbook of Research on Complex Dynamic Process Management: Techniques for Adaptability in Turbulent Environments, p. xxii-22. Hershey, US: IGI Global,

[31] Osterle, H., Fleisch, E., \& Alt, R. (2001). Business networking Shaping collaboration between enterprises. Springer Verlag.

[32] Papazoglou, M.P., Paolo Traverso, Schahram Dustdar, and Frank Leymann (2006): Service-Oriented Computing Research Roadmap. Technical report/vision paper on Service oriented computing European Union Information Society Technologies (IST), Directorate D - Software Technologies (ST)

[33] Preist, C. (2004). A conceptual architecture for Semantic Web services. In Proceedings of the International Semantic Web Conference 2004 (ISWC 2004)

[34] R. Dijkman et al, Service-oriented Design: A Multi-viewpoint Approach, International Journal of Cooperative Information Systems, Vol. 13, No. 4, pp. 337-368, 2004

[35] Roy, J., and A. Ramanujan (2001). Understanding Web services. IT Professional, 3(6), 69-73. doi:10.1109/6294.977775

[36] Stacey, M., \& Unhelkar, B. (2004). Web services in implementation. Paper presented at the 15th ACIS Conference, Hobart, Australia

[37] Van der Aalst, W. M. P., \& vn Heae, K. M. (2002). Workflow management: Models, methods, and systems.Cambridge, MA: MIT Press.

[38] Wang, M., Cheung, W. K., Liu, J., Xie, X., \& Lou, Z. (2006b). E-Service/process composition through multi-agent constraint management. International Conference on Business Process Management (BPM 2006) (LNCS 4102, pp. 274-289).

[39] Workflow Management Coalition. (1995, January). The workflow reference model

[40] W3C (2004), Web Services Architecture, http://www.w3.org/TR/2004/NOTE-ws-arch-20040211/

[41] Zeng, L., Lei, H., Chang, H. (2007). Monitoring the QoS for Web services. In: Krämer, B.J., Lin, K.-J., Narasimhan, P. (eds.) ICSOC. Lecture Notes in Computer Science, vol. 4749, pp. 132-144. Springer

[42] Zhao, X., Liu, C., \& Yang, Y. (2006). Supporting virtual organisation alliances with relative workflows. Paper presented at the 3rd Asia-Pacific Conference on Conceptual Modelling Hobart, Australia

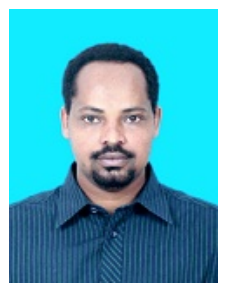

Bekele Teshome Megersa was born in Ethiopia. He obtained his Master of Engineering in software engineering in 2011 from Chongqing University, China and BSc. in computer science from Hawassa University, Ethiopia in 2006. He is currently a Lecturer of Computer Science at the Institute of Technology of Arba Minch University, Ethiopia. He has presented his research findings in the area of business process management and cloud computing at various international conferences and workshops. His research interests include business process management, web services, service oriented architecture and cloud computing. Mr. Bekele is a member IACSIT.

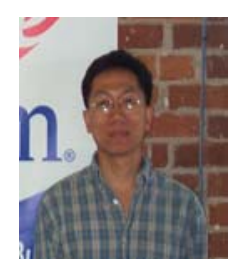

Weihua Zhu was born in China. He got Masters Degree in computer science in 1994 from Louisiana Tech University, Ruston, LA, USA and his BSc. in computer engineering and appliance in 1988 from Chongqing University, China. He has worked as Software Developer, Software Development Consultant and Chief Technology Officer in several USA companies including AT\&T. He has a broad knowledge and experience in the area of e-marketing and service oriented computing. He is currently an Associate Professor and Senior Lecturer and Researcher at college of Software Engineering of Chongqing University, China. He has published several research papers in international conferences and workshops. His research interests include e-business and e-commerce, enterprise information system and image, graphics \& digital media. 